\title{
Reconciling the Evidence of Card and Krueger (1994) and Neumark and Wascher (2000)
}

\author{
Olli Ropponen \\ Government Institute for Economic Research (VATT) and \\ HECER
}

Discussion Paper No. 325

April 2011

ISSN 1795-0562

HECER - Helsinki Center of Economic Research, P.O. Box 17 (Arkadiankatu 7), FI-00014 University of Helsinki, FINLAND, Tel +358-9-191-28780, Fax +358-9-191-28781, E-mail info-hecer@helsinki.fi, Internet www.hecer.fi 


\title{
Reconciling the Evidence of Card and Krueger (1994) and Neumark and Wascher (2000)*
}

\begin{abstract}
We employ the original Card and Krueger (1994) and Neumark and Wascher (2000) data together with the changes-in-changes $(\mathrm{CIC})$ estimator to re-examine the evidence of the effect of minimum wages on employment. Our study reconciles the controversial positive average employment effect reported by the former study and the negative average employment effect reported by the latter study. Our main finding, which is supported by both datasets, is that the controversial result remains valid only for small fast-food restaurants. This finding is accompanied with a new possible explanation.
\end{abstract}

JEL Classification: C21, J23, J38

Keywords: CIC estimator, employment, minimum wage, nonlinear treatment effect models.

Olli Ropponen

Government Institute for Economic Research (VATT)

P.O. Box 1279 (Arkadiankatu 7)

FI-00101 Helsinki

FINLAND

e-mail: olli.ropponen@vatt.fi

* I thank Essi Eerola, Pekka IImakunnas, Tero Kuusi, Tomi Kyyrä, Markku Lanne, Sami Napari, Panu Poutvaara, Heikki Pursiainen, Otto Toivanen and Roope Uusitalo as well as the participants of the workshop in Computational Economics and Econometrics in Helsinki in 2009, the annual meeting of the Finnish Economic Society, the annual meeting of the European Economic Association and the workshop in Labour Economics in Helsinki in 2010 for our discussions. 


\section{INTRODUCTION}

New Jersey experienced an increase in the minimum wage on April 1, 1992. David Card and Alan B. Krueger were the first to use this change to study the employment effect of the minimum wage. They chose Pennsylvania, the neighboring state that did not experience any change in the minimum wage that time, to serve as a control group. The data they collected include observations on fast-food restaurants in both New Jersey and Pennsylvania before and after the minimum wage increase. Card and Krueger's (1994) (CK henceforth) controversial result was that an increase in the minimum wage did not decrease, but as a matter of fact increased overall employment. ${ }^{1}$ This stimulated a lot of discussion on the overall employment effect of the minimum wage. This is still an open issue.

The result was challenged by David Neumark and William Wascher (2000) (NW henceforth). They show that the CK data have more variation than their administrative payroll data, suggesting that the CK data might suffer from an extraordinary amount of measurement error. Their argumentation points to the direction that this measurement error in the telephone survey data employed in CK might have led to false inferences. As the result NW report (p. 1390): "...the payroll data indicate that the minimum-wage increase led to a decline in fast-food FTE employment in New Jersey relative to the Pennsylvania control group." This

\footnotetext{
${ }^{1}$ Before CK several controversial non-negative employment effects of an increase in the minimum wage had already been reported. These studies have exploited variation from both federal (Card 1992a, Lawrence F. Katz and Krueger 1992, Stephen Machin and Alan Manning 1994) and state-specific (Card 1992b) increases in the minimum wage. For the literature concerned with minimum wages we refer to the book by Neumark and Wascher (2008) and for the debate thereafter we refer to an article by Saul D. Hoffman and Diane M. Trace (2009).
} 
is the very opposite to the CK result. Card and Krueger (2000) use in their reply article, the data, which are based on unemployment-insurance payroll-tax records. As the result they report (p. 1419): "The increase in New Jersey's minimum wage probably had no effect on total employment in New Jersey's fast-food industry, and possibly had a small positive effect". This result lies in between the CK and NW results.

Both of these follow up papers as well as most proponents and opponents of the original result have provided additional information via use of new datasets. Another feature most of these studies share is that they are after the average or the total employment effect - a single number. ${ }^{2}$ Our study differs from these by employing the same datasets as CK and NW, but a different estimator. In addition to a point estimate we provide the whole distribution of the employment effects resulting from the New Jersey minimum wage increase. The capability for doing this arises from using the changes-in-changes (CIC) estimator introduced by Susan Athey and Guido W. Imbens (2006) (AI henceforth). The CIC estimator allows for nonlinearities and uses the information on the entire counterfactual distribution instead of just a constant (function). ${ }^{3}$ As we use both the CK and the NW data, our results are not subject to possible measurement errors occurring in the CK data.

\footnotetext{
${ }^{2}$ Some of these have employed quantile difference-in-differences (QDID) estimation, which is capable for going beyond a single number. It has, however, several disadvantages relative to our estimation technique. See Susan Athey and Guido W. Imbens (2006) for a detailed discussion.

${ }^{3}$ We refer to AI for a throughout discussion on the CIC estimator. An excellent review on the development of the literature on program evaluation is provided by Imbens and Jeffrey M. Wooldridge (2009).
} 
Section 2 begins by showing how the counterfactual employment levels are constructed for each New Jersey fast-food restaurant. Using these we then study the employment effects of an increase in the minimum wage in New Jersey. In section 3 we conclude and provide a new potential explanation for the controversial result. ${ }^{4}$

\section{A CASE STUDY OF THE FAST-FOOD INDUSTRY}

New Jersey experienced an increase in the minimum wage on April 1, 1992. By using this state-specific variation we study the employment effects using both the DID and the CIC estimators. The data employed are those in CK and NW. ${ }^{5}$ These panel data include observations on fast-food restaurants in both New Jersey and eastern Pennsylvania before and after the minimum wage increase. ${ }^{6}$ The balanced sample in CK includes observations on the fast-food restaurants with no missing information on employment variables. It has 309 observations on fastfood restaurants in New Jersey and 75 in Pennsylvania, making the total number of observations 384 . We use 376 observations from the balanced sample. We have

\footnotetext{
${ }^{4}$ We have failed to find any paper providing an estimation routine for the CIC estimator in the R-environment. Our R-codes are available upon request. Athey provides one in Matlab language in her homepage. It is employed in the CIC estimation in the supplementary material of AI.

${ }^{5}$ We refer to $\mathrm{CK}$ and NW for the throughout discussions about their data. CK data are available both in http://www.irs.princeton.edu/Links/MinimumWage.php and http://econwww.mit.edu/faculty/angrist/data1/mhe/card. The NW payroll data were provided by Neumark and Wascher.

${ }^{6}$ The CK observations before the increase are collected between February and March 1992 and the ones after the increase between November and December 1992. The NW data contain observations from February and November 1992.
} 
to exclude 8 New Jersey observations in order to meet the identification conditions of the CIC estimator. ${ }^{7}$ The NW data include 235 observations on fast-food restaurants in February $1992 .^{8} 230$ of these, 159 in New Jersey and 71 in Pennsylvania, remained open in November 1992 and are documented in figures 1 and 2 in NW. Again, we must exclude 8 New Jersey observations in order to meet the identification conditions of the CIC estimator and thus work with 222 observations when using the NW data. We follow CK in choosing the measure for employment level to be the full-time equivalent (FTE) employment. It is calculated for the CK data as the sum of the number of managers, the number of full-time workers and half the number of the part-time workers. The NW payroll data include hours worked by nonmanagement employees and are given on a weekly, biweekly or monthly basis. These are first converted to weekly basis ${ }^{9}$ and then divided by 35 - the assumed hours of a full-time workweek - to obtain a measure of FTE employment.

\subsection{Construction of the Counterfactual Employment Levels}

In the treatment effect estimation we are interested in the effect a given "treatment" has on the units being subjected to it. The effect is defined as the difference between the outcome that occurs after the treatment and the one that would have occurred in its absence. As the latter is unobserved we have to come up with the

\footnotetext{
${ }^{7}$ For the excluded restaurants the employment levels before the minimum wage increase are not in the domain of employment levels in Pennsylvania at that time.

${ }^{8}$ The sample characteristics are given in table 2 in NW.

${ }^{9}$ Here we follow NW and take into account the difference in the numbers of days in February and November - and the fact that year 1992 was a leap year.
} 
counterfactual outcomes. The way these are constructed differ between the DID and CIC estimators, and due to this difference the CIC estimator is able to provide us information about the treatment effects beyond the conventional DID estimator. The CIC estimator is able to provide observation-specific treatment effects which are based on a (more) flexible construction of the counterfactual outcomes (than in the case of the conventional DID estimator). This is illustrated by CK data in figure 1.

Let us denote by $G_{i} \in\{0,1\}$ the group (control or treatment) and by $T_{i} \in$ $\{0,1\}$ the period (before or after) of observation $i$, and by $N_{g t}$ the number of observations in group $g$ in period $t$. Let $Y_{g t, i}$ stand for the outcome of variable $Y$ for observation $i$ in group $g$ in period $t$, and let $F_{Y, g t}$ be the corresponding cumulative distribution function. The estimator for the average treatment effect in this $(\mathrm{AI})$ notation reads as:

$$
\hat{\tau}^{C I C}=\frac{1}{N_{11}} \sum_{i=1}^{N_{11}} Y_{11, i}-\frac{1}{N_{10}} \sum_{i=1}^{N_{10}} \hat{F}_{Y, 01}^{-1}\left(\hat{F}_{Y, 00}\left(Y_{10, i}\right)\right),
$$

where $\hat{F}_{Y, g t}$ is the empirical counterpart for $F_{Y, g t}$ - that is the empirical cumulative distribution function. Thus, the average treatment effect is the difference between the averages of the observed outcomes of the treatment group in period 1, $Y_{11, i}$, and the counterfactual outcomes for that period, $\hat{F}_{Y, 01}^{-1}\left(\hat{F}_{Y, 00}\left(Y_{10, i}\right)\right)$. With panel data available, we are able to calculate the observation-specific treatment effects, $Y_{11, i}-\hat{F}_{Y, 01}^{-1}\left(\hat{F}_{Y, 00}\left(Y_{10, i}\right)\right)$, as well.

The CIC counterfactuals are constructed in two steps. The upper graphs of figure 1 illustrate the first and the lower ones the second step of the construction of the counterfactual employment level for a New Jersey fast-food restaurant with the FTE employment level of 40 in early 1992 - that is before the minimum 
wage increase. As the first step we identify the quantile this type of New Jersey fast-food restaurant would correspond to if it was in Pennsylvania at that time. The upper left hand side graph plots the empirical cumulative distribution function (ecdf) for FTE employment in Pennsylvania $\left(\hat{F}_{Y, 00}\right.$ using the notation in AI) and the upper right hand side graph plots that in New Jersey $\left(\hat{F}_{Y, 10}\right)$ before the increase in the minimum wage. These show that a fast-food restaurant in New Jersey with an employment level of 40 in early 1992 corresponds to quantile of about 0.95 whereas if it was in Pennsylvania it would correspond to quantile of about $0.90 .{ }^{10}$ The second step includes the determination of the new employment level for the New Jersey fast-food restaurant with FTE employment of 40 in early 1992. It is determined by the evolution of the employment level of the Pennsylvania quantile identified in the first step. Thus, an evolution of the employment level of the fast-food restaurant in New Jersey that had 40 FTE workers before the increase in the minimum wage is supposed, in the absence of the increase, to follow the evolution of the 0.90 quantile in Pennsylvania (even if the New Jersey quantile in the ecdf it originally belongs is at about 0.95). ${ }^{11}$ The lower left hand side graph plots the ecdf's for FTE employment in Pennsylvania both before $\left(\hat{F}_{Y, 00}\right)$ and after $\left(\hat{F}_{Y, 01}\right)$ the New Jersey minimum wage increase. It shows that ${ }^{10} \hat{F}_{Y, 10}(40) \approx 0.95$ and $\hat{F}_{Y, 00}(40) \approx 0.90$.

${ }^{11}$ In QDID estimation one would use the quantile of about 0.95 in determining the counterfactual employment level. In CIC estimation the identification of the quantile in the construction of counterfactual is based on the control group restaurant with the same size. In QDID estimation the corresponding restaurant does not have to be of the same size, but may differ a lot depending on the differences between the distributions of treatment and control group. The QDID estimator gives us the estimate for the average employment effect of 3.10 FTE for CK data and -1.00 FTE for NW data. 
the employment level of the identified quantile has moved from 40 in early 1992 to 34 in late $1992 .{ }^{12}$ This is also taken to be the counterfactual value for the New Jersey fast-food restaurant with FTE employment level of 40 in early 1992.

We repeat the two steps for each of the New Jersey fast-food restaurants with FTE employment levels of $Y_{10}$. Here we first identify the Pennsylvania quantile being followed in determining the counterfactual evolution in time by calculating $\hat{F}_{Y, 00}\left(Y_{10}\right)$. Then we determine the counterfactual values for the identified quantile by calculating $\hat{F}_{Y, 01}^{-1}\left(\hat{F}_{Y, 00}\left(Y_{10}\right)\right)$. The resulting counterfactual employment levels are depicted in the lower right hand side graph in figure 1 together with the ecdf for FTE employment in New Jersey before the minimum wage increase. These are given as a function of initial employment levels in figure $2 .{ }^{13}$

In the case of a continuous variable we get a point estimate for the average treatment effect implied by the CIC estimator by using equation 1 with $\hat{F}_{Y, g t}^{-1}(q)$ defined as

$$
\hat{F}_{Y, g t}^{-1}(q)=\inf \left\{y \in \mathbb{Y}_{g t}: \hat{F}_{Y, g t}(y) \geq q\right\}
$$

This is not true for the discrete variables. In the case of a discrete variable ${ }^{14}$ we get upper and lower bounds for the counterfactual outcomes and therefore

\footnotetext{
${ }^{12} \hat{F}_{Y, 01}^{-1}\left(\hat{F}_{Y, 00}(40)\right)=34$.

${ }^{13}$ The corresponding graph by using the conventional DID estimator would be a straight line with the slope of unity.

${ }^{14}$ The observed FTE employment levels in CK are restricted by the definition to be in discrete intervals. If one treated these as outcomes from a continuous variable, one would be using the upper bounds of the counterfactual outcomes and therefore the results from the lower bounds of the treatment effects. The bounds are said to be tight in p. 453 in AI and in our study the qualitative results would remain the same if we treated the FTE employment as being a continuous variable.
} 
also for the treatment effects. The upper bounds of the counterfactual outcomes are the same as in the continuous case, and for the lower bounds we replace the inverse function $\hat{F}_{Y, g t}^{-1}$ in equation 1 by $\hat{F}_{Y, g t}^{(-1)}$, where

$$
\hat{F}_{Y, g t}^{(-1)}(q)=\sup \left\{y \in \mathbb{Y}_{g t} \cup\{-\infty\}: \hat{F}_{Y, g t}(y) \leq q\right\}
$$

In the following section we provide both upper and lower bounds for the employment effects.

\subsection{Employment Effects in New Jersey}

In figure 3 we plot the ecdf for CK data New Jersey restaurants after the minimum wage increase together with the counterfactual distribution. If the order of the restaurants in the distribution remained unchanged between early 1992 and late 1992, we would be able to read the employment effects for each of the fast-food restaurants directly from the figure. Then the employment effect for, say, the restaurant with FTE employment level of 40 in early 1992 would be about 6 units, because the counterfactual distribution evolved to 34. The employment effect would in this case simply be the horizontal difference between the late 1992 curve and the counterfactual. For New Jersey the order of the restaurants in the distribution changes and thus this special condition is not met. In addition, we are not only interested in the distributional change, but also in the restaurantspecific employment effects. ${ }^{15}$ These can be evaluated by comparing the actual outcomes to the counterfactual outcomes in New Jersey in late 1992 for each restaurant. For example an employment level of a fast-food restaurant in New

\footnotetext{
${ }^{15}$ Panel data enable us to study the restaurant-specific employment effects, whereas with cross-sectional data we would be restricted to distributional changes only.
} 
Jersey with early 1992 employment level of 40 should have evolved to 34 and the employment effect is considered to be the difference between the true employment outcome of this fast-food restaurant and 34 . If the true outcome is, say 25 , then the employment effect for that restaurant would be negative with a decrease of 9 units in the FTE employment.

If we were purely interested in changes in the distribution, we could just calculate the employment effects for each of the quantiles. This approach is unfortunately somewhat restricted. Suppose that the distributions in both New Jersey and Pennsylvania were the same before and after the minimum wage increase, but in New Jersey two fast-food restaurants had changed places in the distribution from early 1992 to late 1992. Quantile-specific employment effects would in this case be zero for all the quantiles, whereas the fast-food restaurant-specific employment effects would all be zero except for the two fast-food restaurants - the ones that change the place in the distribution. One of these is affected positively by the minimum wage increase whereas the other is affected negatively. This does not show up when calculating quantile-specific employment effects. Despite the differences, these two ways result in the same average employment effect.

In figure 4 we plot the upper bounds for the employment effects for each of the CK fast-food restaurants in New Jersey together with the DID estimate and the upper bound for the average employment effect using the CIC estimator as well as the smoothed dependence for the conditional average employment effects. The DID estimate for the change in the average FTE employment is $\hat{\tau}^{D I D}=$ 2.72. This corresponds to $\hat{\tau}^{D I D}=2.75$ reported in CK for a balanced sample of restaurants in their table 3 and differs from it due to eight excluded restaurants. 
The upper bound for the average employment effect implied by the CIC estimator is $\hat{\tau}^{C I C}=1.70$. This is calculated as the average of the employment effects for the individual fast-food restaurants. As the CIC estimator is able to provide us restaurant-specific employment effects, it allows us to study the employment effects in more detail. In our case it allows us to study the conditional average employment effects of the change in the minimum wage. These are calculated here by using the lowess smoothing procedure ${ }^{16}$ across the restaurant-specific employment effects. The resulting curve is positive for small fast-food restaurants and turns negative for big fast-food restaurants.

Figure 5 corresponds to figure 4 when using the NW data. The DID estimate for the change in the average FTE employment is $\hat{\tau}^{D I D}=-0.59$ and the upper bound for the average employment effect implied by the CIC estimator is $\hat{\tau}^{C I C}=-1.22^{17}$. The smoothed dependence that represents the conditional average employment effects provides the very same pattern as in the case of CK data: the employment effect is positive for small fast-food restaurants and negative for big fast-food restaurants.

Figure 6 plots the corresponding graphs for figures 4 and 5 for the lower bounds. First, it shows that the conditional average employment effects resemble the ones in figures 4 and 5 - that is the employment effect is typically positive

\footnotetext{
${ }^{16}$ The lowess procedure uses locally-weighted polynomial regression. We use this procedure because the behavior of local polynomial regression procedures at the boundary is often superior to kernel and spline estimation (see Adonis Yatchew, 2003, for a detailed discussion about semiparametric regression methods and its section 3.4 for details about the local polynomial smoothers).

${ }^{17}$ The bootstrap standard error is 0.27 .
} 
for small fast-food restaurants and negative for big fast-food restaurants. Despite the qualitative similarity, some quantitative differences are observed as the employment effect turns from positive to negative at 24 FTE employment in case of CK data and at 14.4 in case of NW data. Therefore, for the fast-food restaurants with the FTE employment level between 14.4 and 24, the results based on CK data suggest a positive effect, whereas NW data suggest a negative effect. This difference, however, vanishes when we take control variables into account (see figure 7). Second, it shows that the biggest estimated employment effects are around 30 in CK data and around -10 in NW data. The absolute value of the first one is about three times that of the latter one and the variances of the estimated employment effects are 57.9 and 14.6 for $\mathrm{CK}$ and NW data respectively. Thus, the estimated employment effects using the CK data exhibit more variation than the ones using the NW data. This is also the case for the original FTE employment levels, for which the variances are 68.3 and 21.4 respectively. The DID estimates of the change in the average FTE employment using the CK and the NW data are $\hat{\tau}^{D I D}=2.72$ and $\hat{\tau}^{D I D}=-0.59$, and the lower bounds for the average employment effect implied by the CIC estimator are $\hat{\tau}^{C I C}=0.90^{18}$ and $\hat{\tau}^{C I C}=-1.82$.

We study the employment effects of an increase in the minimum wage also with control variables in order to check the robustness of our results. The CIC estimation with the control variables, $X$, is done in three steps. First, we estimate the regression

$$
Y_{i}=D_{i}^{\prime} \delta+X_{i}^{\prime} \beta+\epsilon_{i},
$$

\footnotetext{
${ }^{18}$ The bootstrap standard error is 0.44 .
} 
where $D=((1-T)(1-G),(1-T) G, T(1-G), T G)$ in order to get estimates for $\delta$ and $\beta$. Second we construct the residuals with the group-time effects left in:

$$
\hat{Y}_{i}=Y_{i}-X_{i}^{\prime} \hat{\beta}=D_{i}^{\prime} \hat{\delta}+\hat{\epsilon}_{i}
$$

and finally we apply the CIC estimator to $\hat{Y}_{i}$.

In figure 7 , which corresponds to figures 4-6 with control variables, we plot the upper and lower bounds for the employment effects using the CK and NW data. ${ }^{19}$ Each of the graphs provides the employment effects for each of the fastfood restaurants in New Jersey together with the average employment effects using both DID and CIC estimators as well as a smoothed dependence for the conditional average employment effects. ${ }^{20}$ The result the graphs provide is the very same as those without control variables: the employment effect is positive among small fast-food restaurants and negative among big fast-food restaurants.

\section{CONCLUSIONS}

David Card and Alan B. Krueger impugned an old consensus on the overall employment effect of the minimum wage in their paper in 1994 (CK). That article as well as their book in 1995 and the reply article in 2000 share the conclusion that it is highly unlikely that the increase in New Jersey's minimum wage in 1992 would have had a negative effect on the total employment in its fast-food industry.

\footnotetext{
${ }^{19}$ For CK data we control for chain, company-ownership and location. For NW data we control for chain and company-ownership.

${ }^{20}$ The figure shows the results using $301 \mathrm{CK}$ (151 NW) observations on fast-food restaurants in New Jersey and 75 (71) in Pennsylvania.
} 
In contrast, David Neumark and William Wascher conclude in their paper in 2000 (NW) and in their book in 2008 that the average employment effect is likely to be negative. By employing both CK and NW data we have studied the employment effects of the fast-food restaurants conditional on their employment levels using a more flexible estimator than the previous authors. Both datasets lead to the conclusion that these conditional employment effects are positive for small and negative for big fast-food restaurants. Thus, the controversial result in CK is overturned for big fast-food restaurants and the NW data are shown to provide evidence of a positive employment effect for the small fast-food restaurants.

Monopsonistic labor market models might provide an explanation for the observed positive employment effect (see e.g. Tito Boeri and Jan van Ours, 2008). These models are ruled out in CK due to their incapability of explaining pricing behavior. We also rule these models out, but for a different reason. One particular implication of the monopsonistic labor market models is that the employment effect is increasing with respect to the employment level. This is in sharp contrast with our results.

Our results suggest a new explanation, based on the location of restaurants and a demand side effect: An increase in the minimum wage increased spending by those people, who used to earn less than the new minimum wage. This additional spending increased the demand for the restaurant services and resulted in a positive employment effect. This would explain our results if small restaurants are predominantly in poor neighborhoods and large restaurants in areas where close to all of the demand comes from customers earning more than minimum wage. 


\section{REFERENCES}

Athey S, Imbens GW. 2006. Identification and Inference in Nonlinear Difference-in-Differences Models. Econometrica 74: 431-497.

Boeri T, van Ours J. 2008. The Economics of Imperfect Labor Markets. Princeton University Press, Princeton NJ.

Card D. 1992a. Using Regional Variation in Wages To Measure the Effects of the Federal Minimum Wage. Industrial and Labor Relations Review 46: $22-37$.

Card D. 1992b. Do Minimum Wages Reduce Employment? A Case Study of California, 1987-89. Industrial and Labor Relations Review 46: 38-54.

Card D, Krueger AB. 1994. Minimum Wages and Employment: A Case Study of the Fast-Food Industry in New Jersey and Pennsylvania. The American Economic Review 84: 772-793.

Card D, Krueger AB. 1995. Myth and Measurement. Princeton University Press, Princeton NJ.

Card D, Krueger AB. 2000. Minimum Wages and Employment: A Case Study of the Fast-Food Industry in New Jersey and Pennsylvania: Reply. The American Economic Review 90: 1397-1420.

Hoffman SD, Trace DM. 2009. NJ and PA Once Again: What Happened to Employment When the PA-NJ Minimum Wage Differential Disappeared? The Eastern Economic Journal 35: 115-128.

Imbens GW, Wooldridge JM. 2009. Recent Development in the Econometrics of Program Evaluation. Journal of Economic Literature 47: 5-86. 
Katz LF, Krueger AB. 1992. The Effect of the Minimum Wage on the Fast Food Industry. Industrial and Labor Relations Review 46: 6-21.

Machin S, Manning A. 1994. The Effects of Minimum Wages on

Wage Dispersion and Employment: Evidence from the U.K. Wage Councils. Industrial and Labor Relations Review 47: 319-29.

Neumark D, Wascher W. 2000. Minimum Wages and Employment: A Case Study of the Fast-Food Industry in New Jersey and Pennsylvania: Comment. The American Economic Review 90: 1362-1396.

Neumark D, Wascher W. 2008. Minimum Wages. The MIT Press, Cambridge, Massachusetts, London, England.

Yatchew A. 2003. Semiparametric Regression for the Applied Econometrician. Cambridge University Press, Cambridge, United Kingdom. 

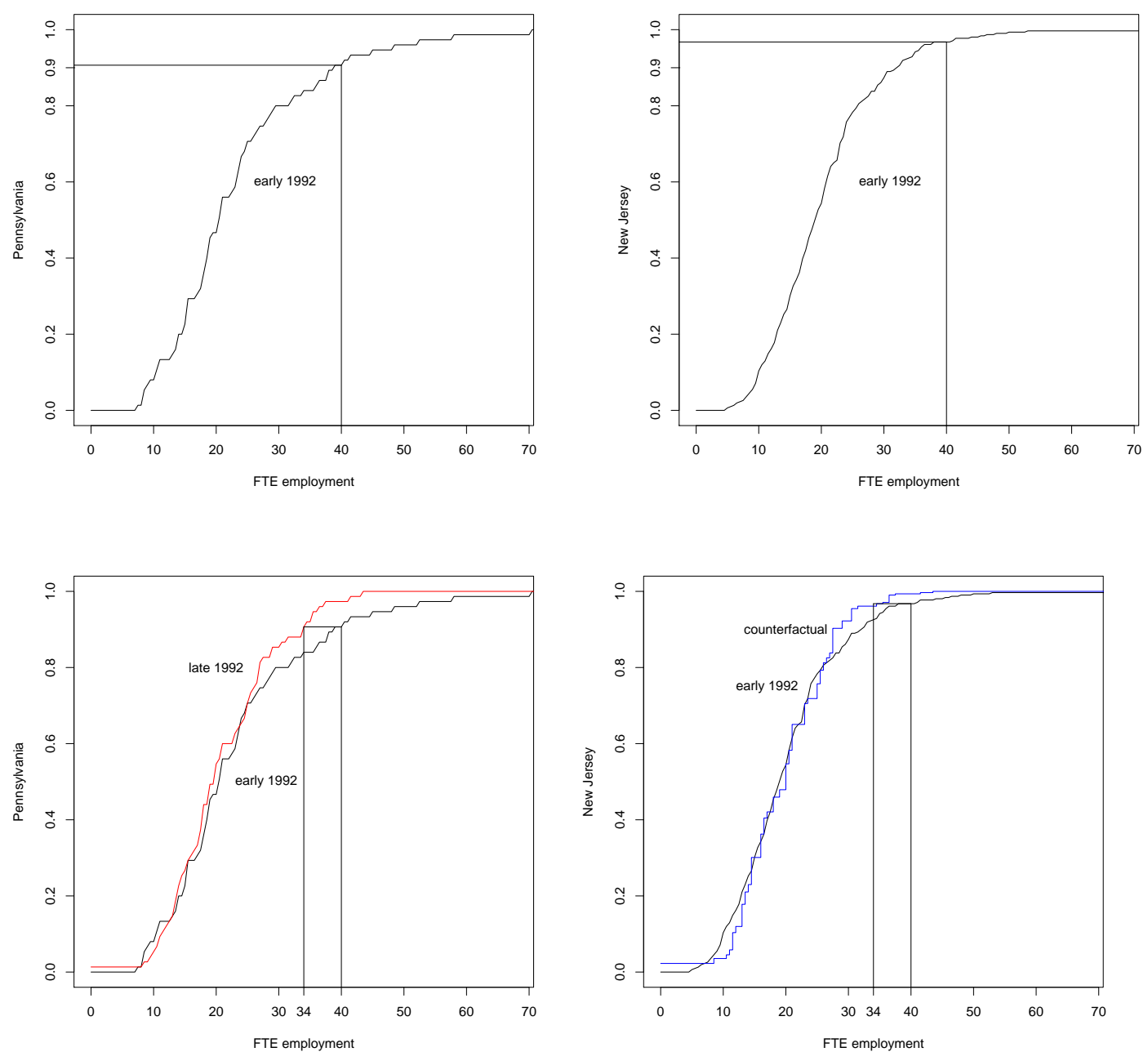

Figure 1: The construction of the counterfactual employment level for the CK data New Jersey fast-food restaurant with FTE employment level of 40 in early 1992. The upper graphs plot the empirical cumulative distribution functions (ecdf) for the FTE employment in Pennsylvania (left hand side graph) and New Jersey (right hand side graph) in early 1992. For the lower graphs we add the ecdf for the FTE employment in Pennsylvania in late 1992 (left hand side graph) and the ecdf for the counterfactual FTE employment in New Jersey (right hand side graph). 




Figure 2: The counterfactual employment for the CK data as a function of FTE employment before the minimum wage increase. 


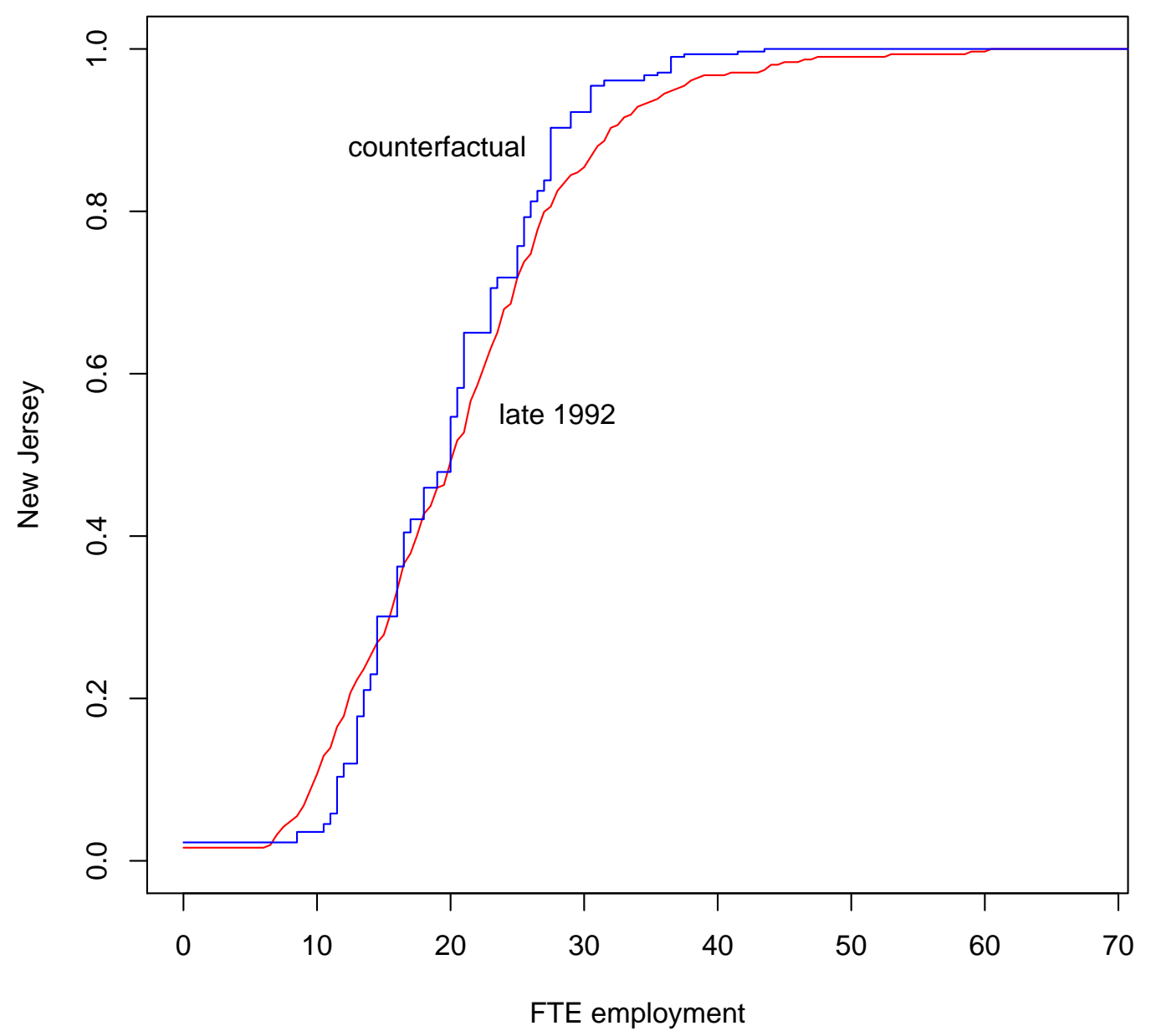

Figure 3: The empirical cumulative distribution function for New Jersey after the minimum wage increase together with the counterfactual distribution for the CK data. 


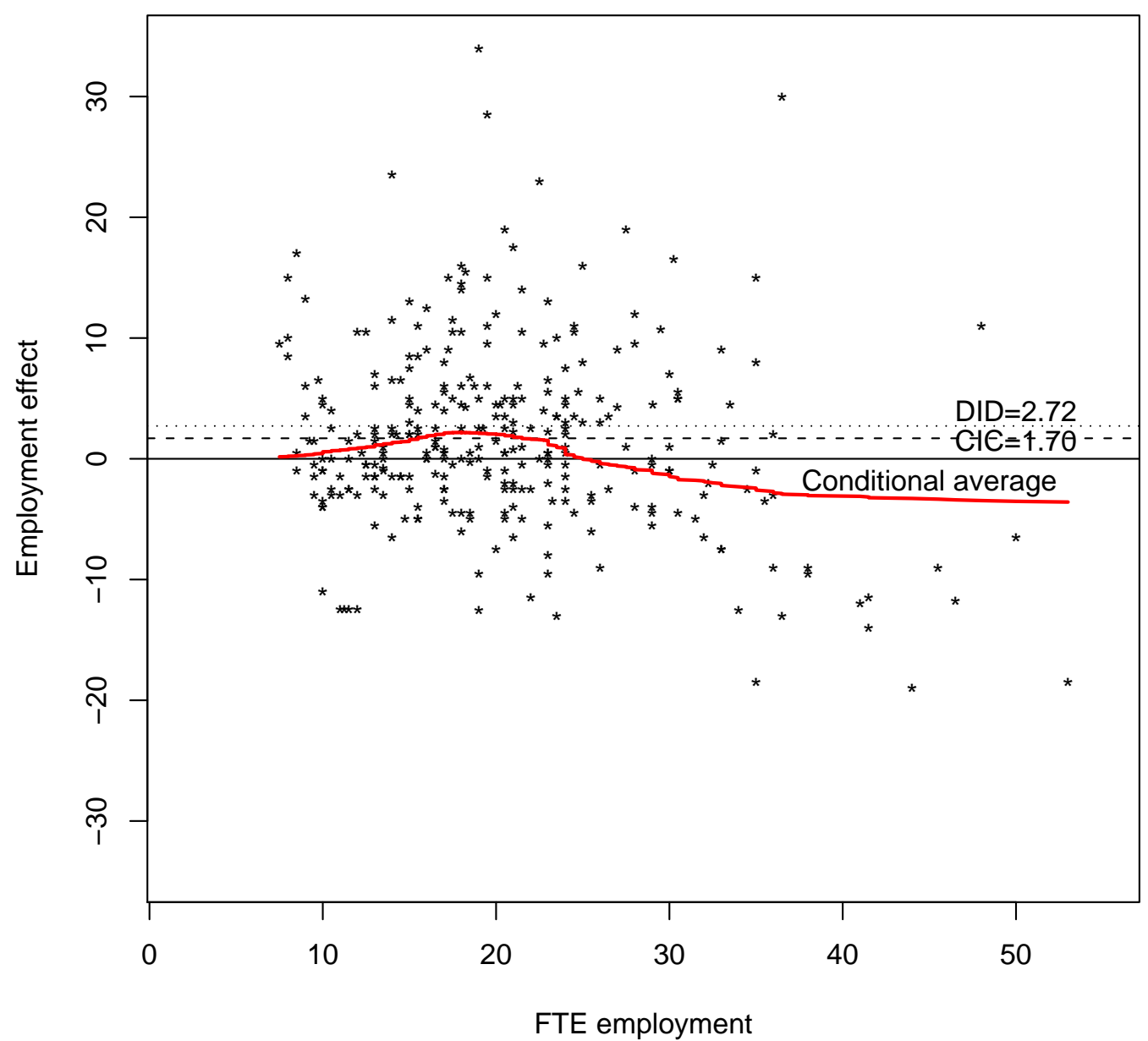

Figure 4: The upper bounds for the employment effects for each of the fastfood restaurants in New Jersey $(*)$ together with the DID estimate (dotted line) and the upper bound for the average employment effect using the CIC estimator (dashed line) as well as a smoothed dependence for the conditional average employment effects (solid curve) when using CK data. 


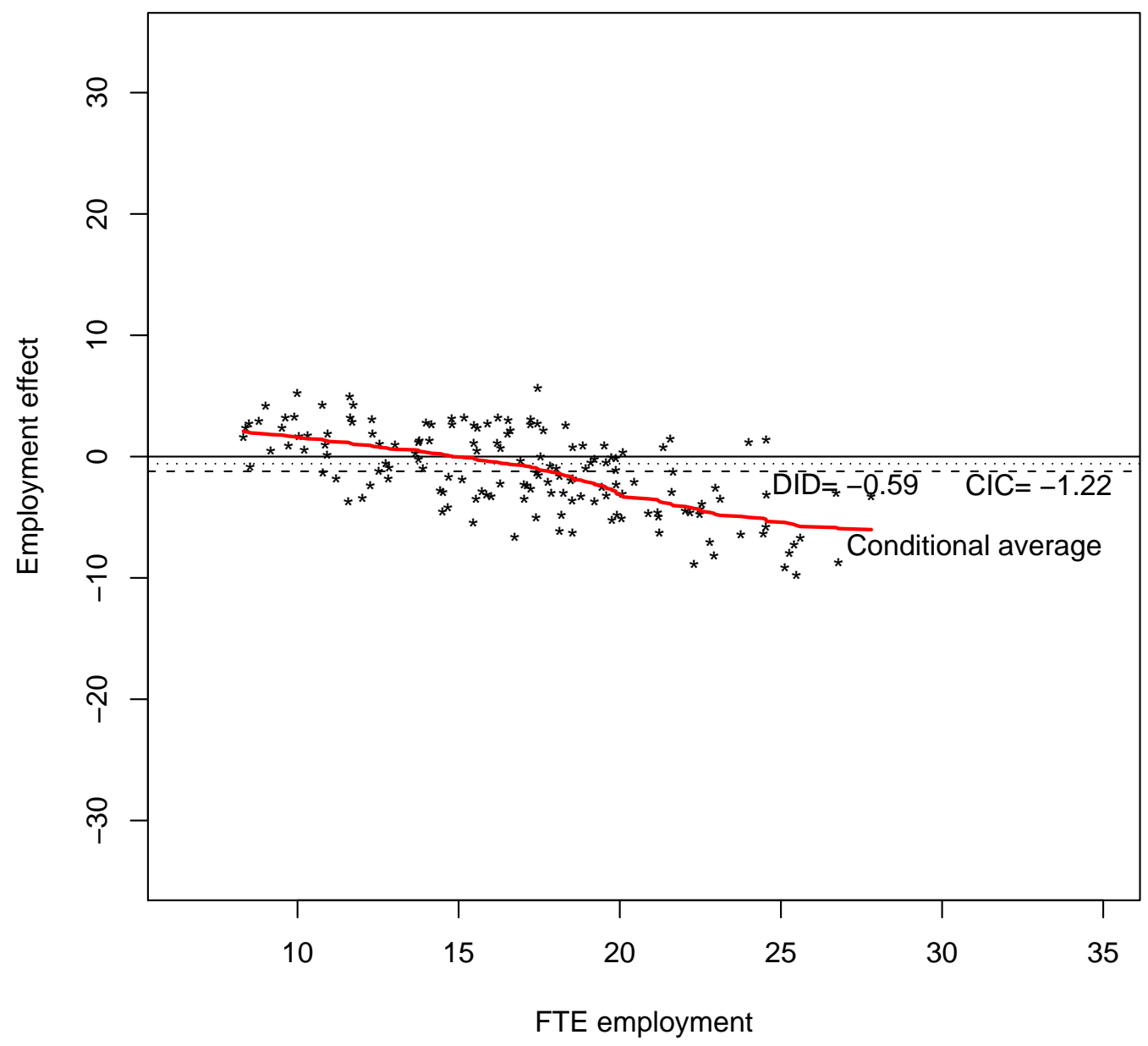

Figure 5: The upper bounds for the employment effects for each of the fastfood restaurants in New Jersey $(*)$ together with the DID estimate (dotted line) and the upper bound for the average employment effect using the CIC estimator (dashed line) as well as a smoothed dependence for the conditional average employment effects (solid curve) when using NW data. 

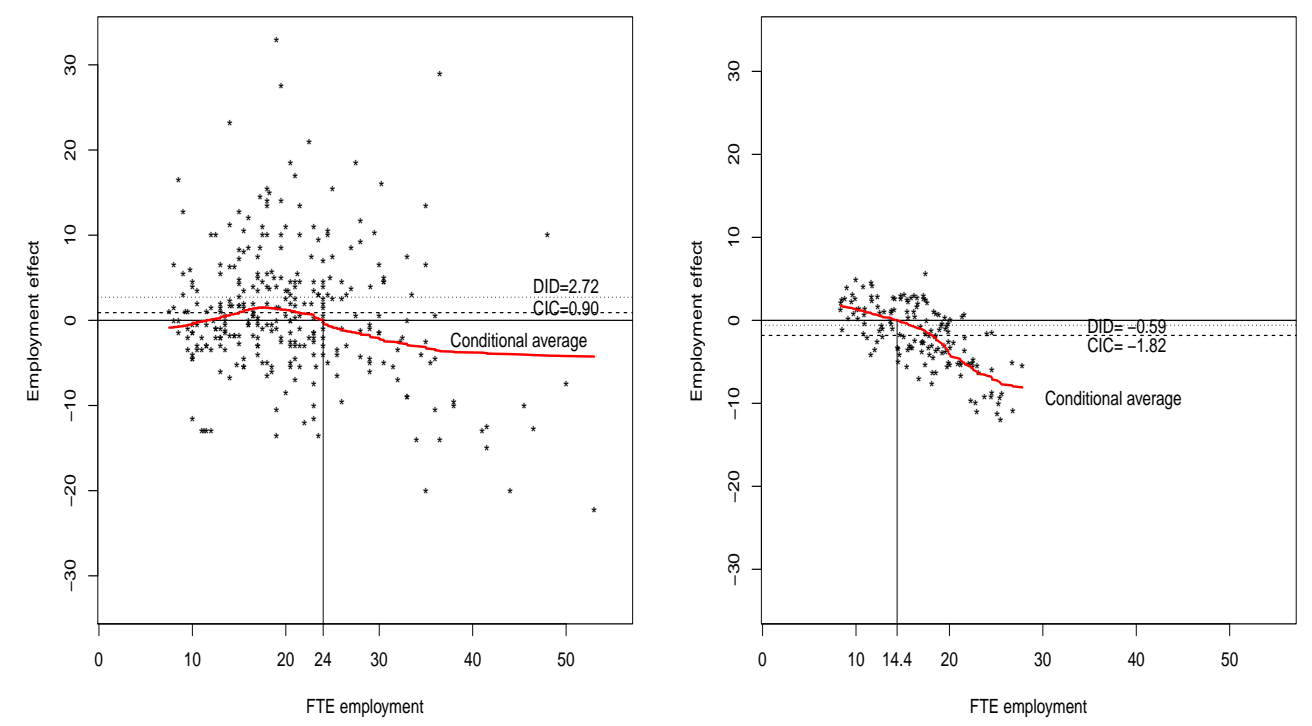

Figure 6: The lower bounds for the employment effects for each of the fast-food restaurants in New Jersey $(*)$ together with the DID estimates (dotted lines) and the lower bounds for the average employment effect using the CIC estimator (dashed lines) as well as a smoothed dependence for the conditional average employment effects (solid curves) when using CK data (left hand side graph) and NW data (right hand side graph). 

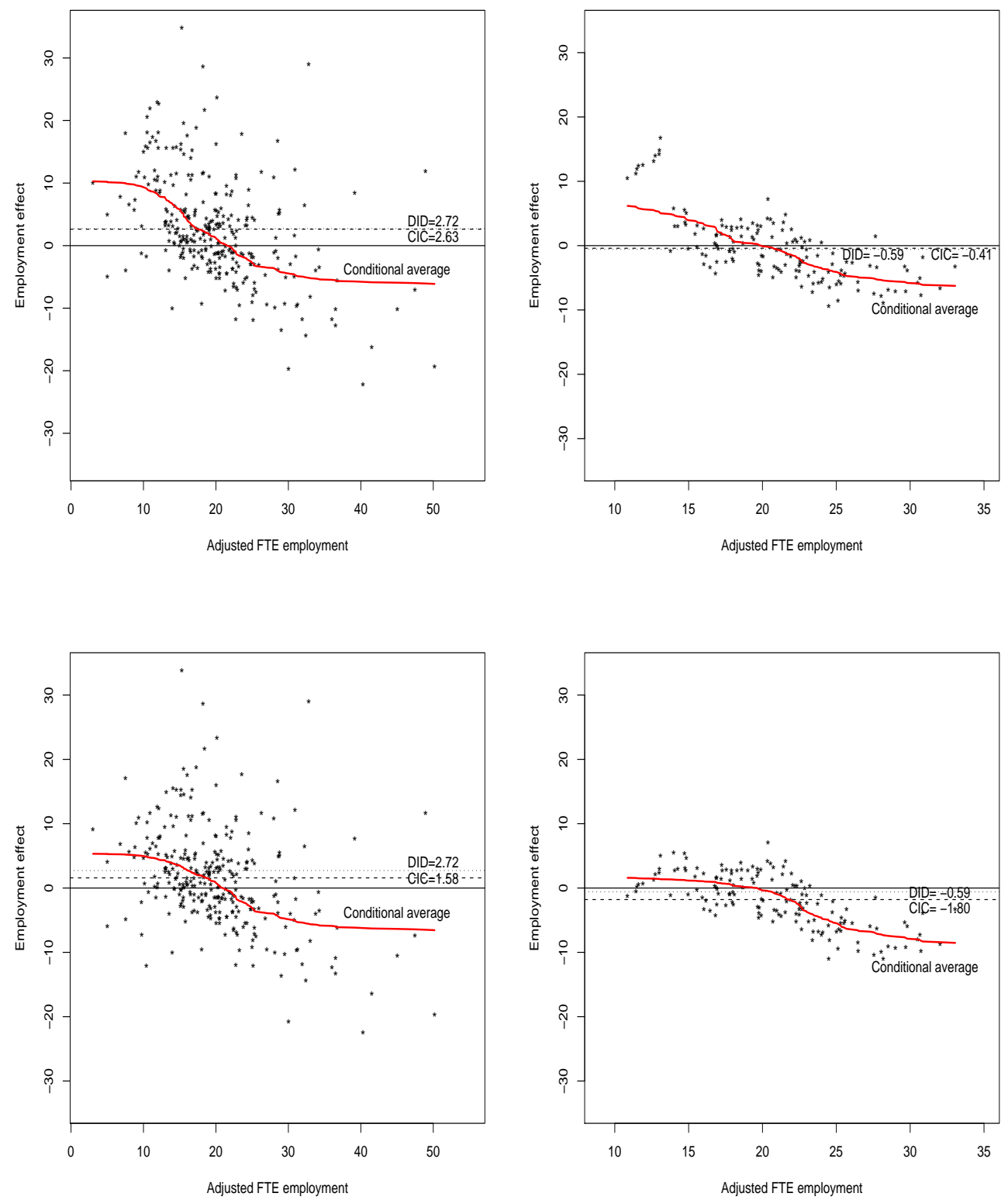

Figure 7: The upper bounds (upper graphs) and the lower bounds (lower graphs) for the employment effects by using CK data (left hand side graphs) and NW data (right hand side graphs) when using control variables. Each of the graphs provides employment effects for each of the fast-food restaurants in New Jersey $(*)$ together with the average employment effects using both DID (dotted line) and CIC estimators (dashed line) as well as a smoothed dependence for the conditional average employment effects (solid curve). 